\title{
Resiliencia y la Intención empresarial en trabajadores peruanos: rol mediador del liderazgo transformacional
}

\author{
Resilience and Entrepreneurial Intention in Peruvian Workers: Mediating \\ Role of Transformational Leadership
}

\author{
Jean A. Ortiz Ramirez ${ }^{1, a}$, Marcos A. Sánchez-Pachas ${ }^{1, b}$, Carlos J. Bardon- \\ Barbieri $^{1, \mathrm{c}}$, Lynncolf E. Toscano-Ortiz ${ }^{1, \mathrm{~d}}$, Sharon J. Rodriguez-Alarcón ${ }^{1, \mathrm{e}} \mathrm{y}$ \\ Iván.S. Leonardo-León León ${ }^{1, f}$ \\ Universidad Nacional Mayor de San Marcos
}

Recibido: $04-10-21$

Aceptado: $29-01-21$

Publicado: $23-12-21$

\section{Resumen}

El objetivo general de este estudio fue evaluar y discutir el papel mediador del liderazgo transformacional entre la asociación de resiliencia y la intención empresarial, en adultos peruanos. En esta investigación participaron 199 adultos, 96 (48\%) mujeres y 103 (52\%) hombres, de edades entre los 18 y 50 años $(M=24, D E=6.94)$. Se evaluaron sus propiedades psicométricas de los instrumentos, obteniendo valores apropiados correspondiente a la evidencia de validez basado en la estructura interna (mediante AFC) y confiabilidad (mediante el método de consistencia interna, coeficiente alfa y omega). El nivel de Liderazgo Transformacional se evaluó con el cuestionario multifactorial MLQ-5X, la resiliencia se evaluó con la escala de Resiliencia de Connor-Davidson y, por último, la intención empresarial fue evaluado mediante el Cuestionario de Intención Empresarial. Los resultados corroboran que a partir de la estimación del efecto mediador del Liderazgo Transformacional entre la resiliencia y la intención empresarial los resultados obtenidos representó $71.1 \%$ de mediación. El efecto directo entre resiliencia sobre la intención empresarial no fue estadísticamente significativo ( $\mathrm{p}=.169,28.9 \%$ ). En conclusión, el liderazgo transformacional cumple el rol mediador de tipo completa, como principal incidencia sobre la variable

1 Escuela de Psicología Organizacional y de la Gestión Humana -UNMSM, Lima, Perú.

a Autor para correspondencia: jeanaxel.ortiz@unmsm.edu.pe ORCID: $\underline{\text { https://orcid.org/0000-0002-7387-9859 }}$

b E- mail: $\underline{\text { marcos.sanchez2@unmsm.edu.pe ORCID: https://orcid.org/0000-0003-3175-9813 }}$

c E-mail: carlos.bardon1@unmsm.edu.pe ORCID: https://orcid.org/0000-0002-8529-9253

d E-mail lynncolf.toscano@unmsm.edu.pe ORCID: https://orcid.org/0000-0002-3174-0111

e E-mail: sharon.rodriguez@unmsm.edu.pe ORCID: $\underline{\text { https://orcid.org/0000-0002-3432-6426 }}$

f E-mail: $\underline{\text { ivan.leon2@unmsm.edu.pe ORCID: https://orcid.org/0000-0001-6772-663X }}$

(C) Los autores. Este artículo es publicado por la Revista de Investigación en Psicología de la Facultad de Psicología, Universidad Nacional Mayor de San Marcos. Este es un artículo de acceso abierto, distribuido bajo los términos de la licencia Creative Commons Atribución 4.0 Internacional (CC BY 4.0) [https://creativecommons.org/licenses/by/4.0/deed.es] que permite el uso, distribución y reproducción en cualquier medio, siempre que la obra original sea debidamente citada de su fuente original. 
intención empresarial. Por último, hay evidencia de una fuerza predominante del liderazgo transformacional como variable mediadora.

Palabras clave: Adultos; resiliencia; liderazgo transformacional; intención empresarial.

\begin{abstract}
The general objective of this study was to evaluate and discuss the mediating role of transformational leadership between the association of resilience and entrepreneurial intention in peruvian adults. A total of 199 adults, $96(48 \%)$ women and 103 (52\%) men, aged between 18 and 50 years $(M=24, S D=6.94)$ participated in this research. The psychometric properties of the instruments were evaluated, obtaining appropriate values corresponding to the evidence of validity based on internal structure (by means of AFC) and reliability (by means of the internal consistency method, alpha and omega coefficient). The level of Transformational Leadership was assessed with the MLQ-5X multifactor questionnaire, resilience was assessed with the Connor-Davidson Resilience scale and, finally, entrepreneurial intention was assessed with the Entrepreneurial Intention Questionnaire. The results corroborate that from the estimation of the mediating effect of Transformational Leadership between resilience and entrepreneurial intention the results obtained represented $71.1 \%$ mediation. The direct effect of resilience on entrepreneurial intention was not statistically significant $(\mathrm{p}=.169,28.9 \%)$. In conclusion, transformational leadership plays a complete mediating role, as the main impact on the entrepreneurial intention variable. Finally, there is evidence of a predominant strength of transformational leadership as a mediating variable.
\end{abstract}

Keywords: Adults; resilience; transformational leadership; business intentions. 
El emprendimiento es definido como la capacidad de un individuo para poder plantear y aplicar ideas innovadoras y soluciones creativas, además de estar acompañada de una actitud de pasión y energía (Romero, 2010). De forma más específica, Gonzales y Morales (2012) afirman que el emprendedor es inventor y empresario a la vez, debido a su característica innovadora y a su intención de involucrarse con una empresa. A ello se le suma que ambos autores indican que el principal objetivo del emprendedor es satisfacer desafíos personales o sociales, más allá de la creación de una empresa. Kruger et al. (2000) plantean que la decisión de emprender puede considerarse principalmente voluntaria y consciente. A partir de ello, Liñan y Chen (2006) afirman que la intención empresarial o emprendedora sería el primer paso en el proceso de emprendimiento, a su vez, es esta intención la que posteriormente define los comportamientos emprendedores.

Parra et al. (2017) afirman que la actitud emprendedora es la capacidad de un individuo para poder desarrollar una nueva empresa, a partir de la inspiración, confianza en sí mismo y la valentía para actuar a fin de alcanzar sus objetivos. Específicamente, los mismos autores indican que una actitud emprendedora conlleva una actitud de desear cambiar las cosas, voluntad y valentía para construir un nuevo y propio proyecto, darles sentido a las pequeñas cosas, entre otras. En relación, Koch-Tovar (2012) plantea una lista de características del comportamiento emprendedor, entre ellas, resaltamos la persistencia, la exigencia de eficiencia y calidad, el cumplimiento y la búsqueda de oportunidades.

Según un estudio de Moran, se entiende por resiliencia como una habilidad para mantenerse sano y estable, tanto a nivel físico como psicológico después de situaciones adversas o experiencias traumáticas (Gínez-Silva et al., 2019). De manera general, la resiliencia incorpora áreas como la adaptación interna y externa e incluso se le suele incluir indicadores de salud y bienestar emocional. En ocasiones se ha considerado a la resiliencia como una habilidad propia solo de unos cuantos individuos, sin embargo, se ha demostrado que esta habilidad es un proceso común entre personas que han pasado por situaciones adversas (Uriarte Arciniega, 2005). Una persona resiliente considera que su actuación puede modificar la situación en la que se encuentre, entiende que las decepciones o fracasos son inevitables, pero a su vez son superables (Kharrazi, 2018). Según Silva (2018), contar con una actitud resiliente trae consigo beneficios a nivel psíquico y físico, regulando nuestro estado de ánimo en general como la salud al evitar estrés y ansiedad, además de ser un aprendizaje constante por las diversas situaciones afrontadas.

Dada las variables, según Saavedra (2011); existen ciertos factores que estimulan la formación de actitudes resilientes en las personas mediante la influencia del entorno como la confianza, optimismo, autonomía, sentido de esperanza, autoestima e independencia. Si bien no se puede afirmar que estos factores determinan o desencadenan la resiliencia en las personas, se puede mencionar que incrementa las probabilidades de originar actitudes resilientes en adultos en general. 
Otro estudio de Curbelo y Ziglio (2020) en el que aseguran que debido a la dramática situación y al impacto del nuevo contexto actual, existe una mayor conciencia sobre la resiliencia entre las distintas naciones. Resulta fundamental contar con esta habilidad para poder sobrellevar esta crisis global y a su vez alcanzar los objetivos del desarrollo sostenible (ODS).

Dada la implicancia y novedad del tema de investigación es relevante entender las variables que condicionan o median el comportamiento emprendedor guiado por la resiliencia. En ese sentido, Fernández (2017) en un estudio de investigación, determinó una fuerte presencia de las características de los líderes transformacionales en las personas emprendedoras, esto último en referencia a su capacidad de creación de negocios e implementación ante cambios en su entorno. La resiliencia también juega un rol fundamental en el emprendimiento, ya que como indica Schneider (2007) una persona resiliente es quien además de anteponerse a posteriores tendencias motiva a las demás personas a plantearse un nuevo repertorio de probabilidades, asimismo desarrolla la capacidad para que otros puedan identificar nuevos líderes y esforzarse en ser los mejores.

El liderazgo le hace falta de la resiliencia según Orts (2010) citado por Zúñiga (2017) ya que el líder es aquel individuo que se caracteriza por poseer la habilidad de influir en otros, pero para esto debe contar con las herramientas necesarias. Por esa razón, se debe reforzar los aspectos que actúan de forma resiliente en cada persona, fortaleciendo de esta forma el desarrollo de la toma de decisiones, la autoestima y las relaciones interpersonales además de tener un sentido y significado.

Esta variable en cuestión ha sido definida como aquel que motiva a los subordinados y colaboradores a realizar más de lo que inicialmente se esperaba de ellos con la finalidad de desarrollar sus capacidades y conseguir su implicación (Bass et al., 2003) y; por su parte Masten (2014), Pacico y Batianello (2014) y Yunes $(2003,2015)$ definieron la resiliencia como un proceso dinámico de desarrollo que permite a las personas y comunidades el poder superar de manera saludable y reorientar situaciones adversas o de sufrimiento. La resiliencia siendo parte de la literatura del emprendimiento se puede identificar a través de dos principales categorías: autores que favorecen el concepto de rasgo o habilidad y aquellos que plantean la dimensión contextual viendo la resiliencia como una respuesta a una situación difícil o adversa Bernard, Marie y Dubard, Saulo (2016). Al ver diversos estudios (p.ej., Shapero 1982; Villamar y Harold, 2015; Carvajal \& Bonilla, 2020) relacionando la resiliencia con el emprendimiento, visto desde diversos enfoques, genera vacíos o desprovistos sin poder abarcarlo de forma holística, por otro lado, no quita el hecho del rol significativo que toma la resiliencia al momento que un individuo se convierte en un emprendedor. Bernard y Dubard (2016), además de mencionar el papel que desempeñan los componentes de la resiliencia que acentúan el emprendimiento, no excluyen la aparición de otras variables que puedan 
contribuir en la decisión de los individuos en convertirse en un emprendedor, incluido factores externos.

Por lo tanto, es necesario identificar variables mediadoras que permitan ampliar la las escasas investigaciones en literatura peruana sobre la relación entre la resiliencia y la Intención Emprendedora, puesto que, la resiliencia no es una condición suficiente para desencadenar una intención emprendedora, por lo que se debe ampliar estudios relacionados con variables intervinientes; como en este estudio, empleando el liderazgo transformacional en su papel como variable mediadora de tipo completa. La hipótesis abarca un papel mediador con efectos indirectos, por lo que niveles adecuados de resiliencia y liderazgo transformacional harían que la intención emprendedora aumente significativamente (Figura 1).

Los hallazgos de esta investigación contribuyen a fomentar acciones formativas para trabajadores con actitudes emprendedoras. En adición a aspectos importantes como la demostración concluyente de la estructura interna para cada instrumento utilizado en la investigación

\section{Figura 1}

Propuesta de Modelo Medidor

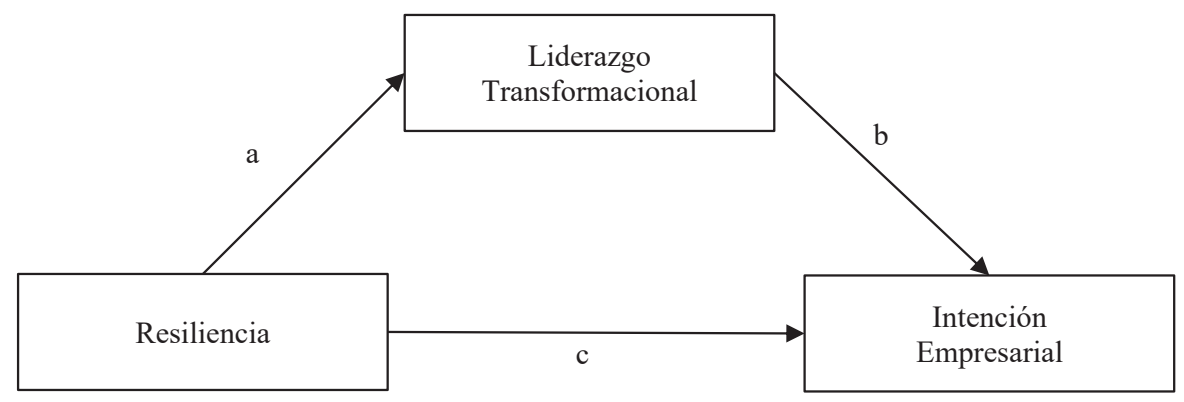

Nota. a: relación Resiliencia y Liderazgo transformacional, b: relación Liderazgo transformacional e Intención empresarial, c: relación Resiliencia e Intención empresarial

En consecuencia, el objetivo general fue evaluar y discutir los efectos mediadores de la variable Liderazgo Transformacional y como objetivos específicos, determinar la relación entre Resiliencia y Liderazgo transformacional, la relación entre Liderazgo transformacional e Intención empresarial y finalmente la relación entre Resiliencia y la Intención empresarial; por consiguiente esta investigación contribuye al limitado conocimiento científico sobre los efectos mediadores existentes entre las variables resiliencia e intención empresarial. Además de impulsar acciones formativas de liderazgo transformacional a individuos con intenciones empresariales. 


\section{MÉTODO}

\section{Diseño}

La investigación de mediación tiene un alcance de tipo explicativo, ya que se propone con el objetivo de probar modelos acerca de las relaciones existentes entre un conjunto de variables (Ato et al., 2013), adopta un enfoque confirmatorio y, además, de acuerdo con las características del presente estudio, se optó considerar un diseño no experimental. Según la clasificación de Salkind (1999), señala que estos estudios se caracterizan por su imposibilidad de manipulación de variables, puesto que mide a las variables en su estado natural.

\section{Participantes}

En este estudio colaboraron un total de 199 personas que trabajaron con un rango mínimo de tres meses durante la pandemia, de los cuales 96 (48\%) fueron mujeres y $103(52 \%)$ hombres cuyas edades oscilan entre 18 y 50 años $(\boldsymbol{M}=24, \boldsymbol{D} \boldsymbol{E}=6.94)$.

\section{Instrumento}

Se utilizó el Cuestionario de Intención Empresarial (E.I.Q.) de Liñan y Chen (2006), el cual es un instrumento que permite medir la intención empresarial de una persona y cuya construcción se basa en el Modelo de Intención Empresarial. Consta de 30 ítems que son medidos en escala de tipo Likert de siete puntos como máximo. Las dimensiones que se utilizaron para evaluar este constructo son: Intención emprendedora y Capacidad emprendedora, las cuales están compuestas por 6 y 3 ítems respectivamente.

El cuestionario de Liderazgo Multifactorial (MLQ-5X) (Bass \& Avolio 1995). Consta de 45 ítems, compuesto por cinco escalas transformacionales, tres transaccionales, una laissez-faire y tres de resultados están incluidos en el MLQ-5X. En consecuencia, para esta investigación se hizo uso de 2 dimensiones de la escala de liderazgo transformacional: Motivación inspiracional, 4 ítems, y Estimulación intelectual, 4 ítems, de tipo Likert con cinco puntos como máximo.

Se usó la escala de resiliencia de Connor-Davidson, en su versión reducida (CD-RISC 10) (Broche, 2012). Esta escala mide únicamente la resiliencia de forma total, consta de 10 ítems de tipo Likert con cinco opciones de respuesta ("en absoluto", "rara vez", "a veces", "a menudo", y "casi siempre"), puntuadas de 1 ("en absoluto") a 5 ("casi siempre"), la cual aumenta en relación directa con las puntuaciones.

Para la estructura interna de los instrumentos aplicados se obtuvo un índice de ajuste global de $\mathbf{C h i} / \mathbf{g l}<5$ (Tabla 1) considerándose adecuado, al mismo tiempo, se observa un índice de ajuste comparativo CFI $\geq .95$, SRMR $<.08$ y RMSEA $<.08$ (Hu \& Bentler, 1999) por lo que los índices de ajustes fueron aceptables, asimismo, 
en la escala de resiliencia (R) se obtuvo Chi/gl (1.7), CFI(.96), SRMR(.04); Liderazgo Transformacional (LT) se obtuvo Chi/gl (2.0), CFI(.97), SRMR(.04) y la Intención Empresarial (IE) se obtuvo Chi/gl (4.6), CFI(.95), SRMR(.04).

Tabla 1

Índice de ajuste de la estructura interna de las variables de estudio $R$, LT y $E(n=199)$

\begin{tabular}{ccccccc}
\hline & $\chi^{2}$ & gl & Chi/gl & CFI & TLI & SRMR \\
\hline R & 58,2 & 34 & 1,712 & 0,96 & 0,95 & 0,04 \\
LT & 38,2 & 19 & 2,011 & 0,97 & 0,96 & 0,04 \\
IE & 121 & 26 & 4,654 & 0,95 & 0,93 & 0,04 \\
\hline
\end{tabular}

Nota: x2; chi cuadrado, gl: grados de libertad; x2/gl: ratio chi cuadrado / grados de libertad, CFI: indice de ajuste comparativo, TLI: indice de Turcker Lewis, SRMR: media cuadrática estandarizada residual, R: Resiliencia, LT: Liderazgo Transformacional, IE: Intención empresarial

En cuanto a la escala de resiliencia $(R)$, liderazgo Transformacional $(L T)$ y la Intención Empresarial (IE) en relación a la confiabilidad por el coeficiente alfa y omega, se puede señalar que la confiabilidad del modelo confirmado resultó aceptable $R(\alpha=.87 ; \omega=.875), L T(\alpha=.872 ; \omega=.875)$ y IE $(\alpha=.952 ; \omega=.952)$.

\section{Procedimiento}

Para evaluar la evidencia de validez basado en la estructura interna de constructo se ha empleado el análisis factorial confirmatorio (AFC) con el uso del programa estadístico Jamovi 1.2, bajo los siguientes criterios: CFI $(\geq .95)$ y TLI $(\geq .90)$ como indicadores de buen ajuste (Hopper et al., 2008) y con valores RMSEA $(\leq .08)$, SRMR $(\leq .08)$ considerados aceptables (Bentler, 1999). Las evidencias de fiabilidad de los instrumentos se obtuvieron a través del método de consistencia interna con los coeficientes $\alpha$ y $\omega \geq .70$ (Frías,2021).

Luego, se estimó la variable de Liderazgo Transformacional como mediador (Baron y Kenny, 1986) entre Resiliencia y la Intención empresarial, utilizando el mismo programa estadístico Jamovi 0.9.5.12, acorde al criterio de que el efecto directo e indirecto se verifica como significativo $(\mathrm{p} \leq .05)$. Por ello, para ser considerado mediador de clase completa o mediación solo indirecta, el efecto indirecto se considera significativo $(\mathrm{p} \leq .05)$, y para una mediación de clase parcial el efecto directo e indirecto se considera no significativo ( $\mathrm{p} \geq .05$ ) (Ato y Vallejo, 2015).

En la investigación se usó para la recolección de datos el Google forms, además se comunicó de forma clara y concreta la finalidad de la investigación, dejando en total libertad el aceptar o rechazar participar voluntariamente de la realización de la encuesta virtual, motivo por el que se consideró agregar un consentimiento informado (Sulivan et al., 2020) en la primera parte del formulario, obteniéndose de esta forma el consentimiento informado con aceptación voluntaria el cual fue difundido a través de diferentes redes sociales. 


\section{RESULTADOS}

\section{Estimación de la mediación}

En relación a los criterios establecidos para el papel mediador (tabla 2, figura 2), se observó que la estimación indirecta $\operatorname{Et}\left(a^{*} b\right)=0.40$, que equivale al $71,1 \%$ ([ $p<$ $.05]$, estadísticamente significativa) de mediación entre la relación de la resiliencia y la intención empresarial. Por otra parte, la estimación directa Et $(c)=.16$ ( $[p>$ $.05], p=.169$ no significativo estadísticamente), equivalente al $28,9 \%$ del efecto directo y; finalmente, con una estimación total de $\mathrm{Et}(c+a * b)=.57$ que evidencia un efecto del 100\%, ([p<.05] es significativo estadísticamente). Es decir, se confirmó una medición de categoría completa o mediación solo indirecta.

Tabla 2

Estimación de mediación

\begin{tabular}{|c|c|c|c|c|c|c|c|c|c|c|}
\hline \multirow[b]{2}{*}{ Efecto } & & & \multirow[b]{2}{*}{ Et } & \multirow[b]{2}{*}{$\mathbf{E}$} & \multirow[b]{2}{*}{$\beta$} & \multicolumn{2}{|c|}{$\begin{array}{l}\text { 95\% Intervalo de } \\
\text { confianza }\end{array}$} & \multirow[b]{2}{*}{$\mathbf{Z}$} & \multirow[b]{2}{*}{$\mathbf{p}$} & \multirow[b]{2}{*}{ \% Mediación } \\
\hline & & & & & & Mínimo & Máximo & & & \\
\hline Indirecto & & & $a * b$ & .409 & - & .25 & .56 & 5,06 & $<.001$ & 71,1 \\
\hline Directo & & & $c$ & .166 & - & -.07 & .40 & 1,37 & 0,169 & 28,9 \\
\hline Total & & & $c+a * b$ & .575 & - & .36 & .78 & 5,37 & $<.001$ & 100 \\
\hline Resiliencia & $\rightarrow$ & $\begin{array}{c}\text { Liderazgo } \\
\text { transformacional }\end{array}$ & $a$ & .411 & .58 & .32 & .49 & 9,92 & $<.001$ & - \\
\hline $\begin{array}{l}\text { Liderazgo } \\
\text { transformacional }\end{array}$ & $\rightarrow$ & $\begin{array}{l}\text { Intención } \\
\text { empresarial }\end{array}$ & $b$ & .995 & .48 & .66 & 1.32 & 5,88 & $<.001$ & - \\
\hline Resiliencia & $\rightarrow$ & $\begin{array}{l}\text { Intención } \\
\text { empresarial }\end{array}$ & $c$ & .166 & .05 & -.07 & .40 & 1,37 & .169 & - \\
\hline
\end{tabular}

Nota: Et: etiqueta, E: estimaciones no estandarizadas, $\beta$ : estimaciones estandarizadas, p: significación estadística, $\%$ M: porcentaje de mediación, a: efecto directo de la Resiliencia y Liderazgo transformacional, b: efecto directo de Liderazgo transformacional e Intención empresarial, c: efecto directo de Resiliencia e Intención empresarial.

\section{Figura 2}

Modelo estructural de las variables de estudio

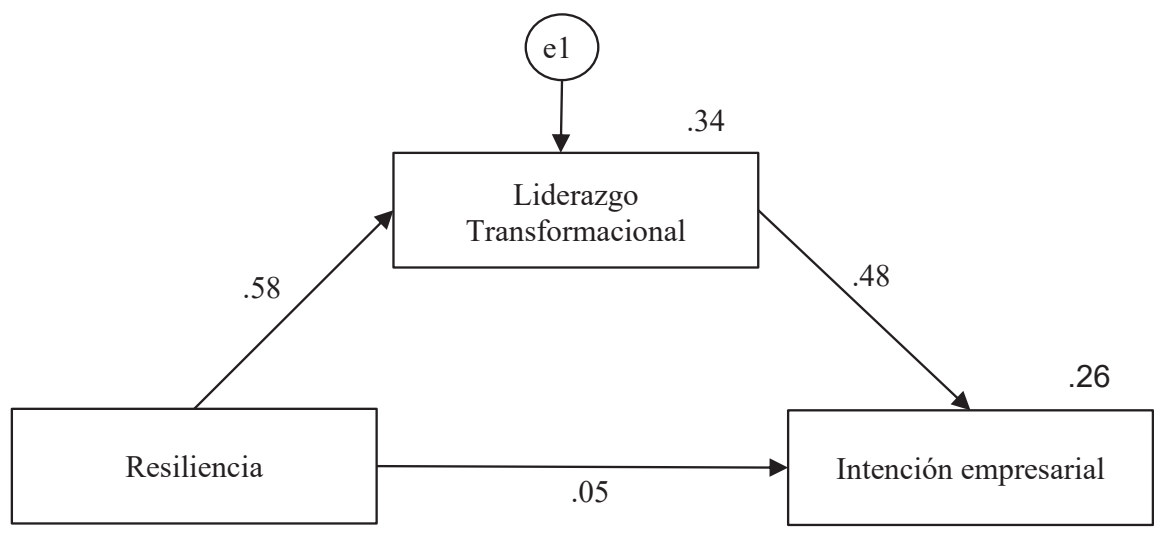




\section{Estimación de los efectos directos}

Con respecto al efecto directo sobre la resiliencia y la intención empresarial (ver figura 1 y tabla 2), el valor estandarizado de $\beta=.05$ se verifica un nivel de significación $p>.05(p=.169)$, a partir de ello, obtenemos que los niveles de resiliencia no generan un efecto directo sobre la Intención empresarial, asimismo el efecto directo de la Resiliencia y el Liderazgo transformacional con valor $\beta=.58$, y el Liderazgo Transformacional sobre la Intención Empresarial con valor $\beta=.48$, estos valores indicando una significancia aceptable con $p<.001$; por lo tanto, la variable resiliencia genera un efecto directo a la variable liderazgo transformacional y la variable Liderazgo transformacional influye directamente en los niveles de Intención Empresarial. Asimismo, el porcentaje de varianza total explicada se debe a la variable Resiliencia y Liderazgo transformacional el cual ha sido del $26 \%$.

\section{DISCUSIÓN}

La investigación en cuestión tuvo como objetivo principal, exponer los efectos mediadores de la variable Liderazgo Transformacional entre resiliencia y la intención empresarial en una muestra de adultos peruanos. Los resultados muestran que la resiliencia causa un efecto indirecto sobre la intención empresarial mientras el Liderazgo transformacional adquiere más significación como mediadora.

En relación al objetivo planteado, los resultados indican que existe un efecto de resiliencia de manera indirecta hacia la intención empresarial. Además, nuestros resultados refieren que existe un efecto directo de la resiliencia sobre el liderazgo transformacional, tal y como Orts (2010) propone al indicar, que un líder necesita poseer un alto grado de resiliencia para poder crear un equipo resiliente.

En adición, nuestro estudio de investigación demuestra que existe un efecto directo de la capacidad de liderazgo transformacional sobre el nivel de intención empresarial de un individuo. Este resultado es congruente con la investigación de Fernández (2017), quién determinó una gran cantidad de características del liderazgo transformacional en las personas emprendedoras.

En relación al impacto de la resiliencia sobre la intención empresarial, según Bernard (2016), el primero tiene un efecto directo sobre el segundo, a sí mismo al ignorar el aspecto procedimental y evolutivo en la trayectoria de la vida del individuo, deja de lado los factores internos como externos que influyen en esta relación, es decir, una literatura incipiente. Sin embargo, cuando se contrasta con una tercera variable interviniente cobra otro panorama, el cual funciona como complemento de lo propuesto por Bernand e indica una relación indirecta a través de la variable mediadora del liderazgo transformacional.

Por otro lado, los resultados obtenidos de su estudio por Carvajal \& Boni1la (2020) concluyen que no necesariamente una persona tiene que ser resiliente 
para generar y mantener una buena idea de negocio, indicando así que no existe correlación entre las variables de resiliencia y emprendimiento de manera directa. No obstante, al no tomar en cuenta el escenario de medición, obvia otro posible resultado; en este caso, el grado de significancia del liderazgo transformacional como variable mediadora y su efecto directo con la intención empresarial que es causalidad directa en el emprendimiento (p.ej., Hernández-López et al.,2018; Escobedo et al., 2020).

Si bien los resultados encontrados en esta investigación representan un preámbulo con fomentar acciones formativas para futuras investigaciones, cabe resaltar la existencia de limitaciones como: El tamaño muestral, el acceso a la muestra, muestreo intencional no probabilístico lo que implica que las interpretaciones tienen que ser realizadas meticulosamente. En consecuencia, no es posible estandarizar o extrapolar estos resultados en cualquier población, es únicamente para la muestra de este estudio.

Sin embargo, esta investigación aporta en presentar una primera evidencia de un modelo mediador; en este caso, el liderazgo transformacional entre la asociación de resiliencia y la intención empresarial en trabajadores peruanos. De ahí que se recomienda para los futuros investigadores seguir verificando en una muestra más grande de tipo probabilístico y no intencional.

\section{Fuentes de financiamiento}

La investigación fue autofinanciada por los propios autores.

\section{Conflicto de intereses}

Los autores declaran no tener ningún conflicto de interés económico, institucional, laboral o personal al realizar el manuscrito.

\section{REFERENCIAS}

Ato, M., \& Vallejo, G. (2011). Los efectos de terceras variables en la investigación psicológica. Anales de Psicología / Annals of Psychology, 27(2), 550-561. https://revistas. um.es/analesps/article/view/123201

Ato, M., López, J. J., \& Benavente, A. (2013). Un sistema de clasificación de los diseños de investigación en psicología. Anales de Psicología / Annals of Psychology, 29(3), 1038-1059. https://doi.org/10.6018/ANALESPS.29.3.178511

Baron, R. M., \& Kenny, D. A. (1986). The moderator-mediator variable distinction in social psychological research: Conceptual, strategic, and statistical considerations. Journal of Personality and Social Psychology, 51(6), 1173-1182. https://doi. org/10.1037/0022-3514.51.6.1173

Bass, B. M., \& Avolio, B. J. (1995). Multifactor Leadership Questionnaire (MLQ) [Database record]. APA PsycTests. https://doi.org/10.1037/t03624-000 
Bernard, M., \& Dubard, S. (2016). Resilience and entrepreneurship: A dynamic and biographical approach to the entrepreneurial act. Management (France), 19(2), 89-123. https://doi.org/10.3917/MANA.192.0089

Broche-Pérez, Yunier \& Rodríguez-Martín, Boris \& Santaella, Susana \& Díaz, Geily \& Carballo, Adelis \& Blanco, Yipsandra. (2012). Escala de Resiliencia de ConnorDavidson (CD-RISC). https://www.researchgate.net/publication/277139516 Escala_de_Resiliencia_de_Connor-Davidson_CD-RISC

Carvajal, Alarcón \& Bonilla-Cruz, Nidia-Johanna \& García, Marisela \& Hernandez, Yurley Karime. (2020). Lineamientos de intervención: resiliencia y emprendimiento en microempresarios del sector turismo en salud. Una innovación psicológica. Archivos Venezolanos de Farmacología y Terapéutica, 38(5), 629-635. https://www.researchgate.net/publication/340464378 Lineamientos de intervencion resiliencia_y emprendimiento en microempresarios_del_sector_turismo en salud Una innovacion_psicologica

Escobedo, R., Véliz, T., Ballón, J., \& Midolo, W. (2020). Emprendimiento empresarial y la intención emprendedora en estudiantes de las Universidades Públicas de la Macro región Sur del Perú. Revista Innova Educación, 2(1), 177-192. https://doi.org/10.35622/j. $\underline{\text { rie.2020.01.011 }}$

Hernández-López, D. M., Moncada-Toro, J. F., \& Henao-Colorado, L. C. (2018). Intención emprendedora de los empleados del sector privado de la ciudad de Medellín y su Área Metropolitana. Revista CEA, 4(8), 13-33. https://doi.org/10.22430/24223182.1045

Escobedo, R., Véliz, T., Ballón, J., \& Midolo, W. (2020). Emprendimiento empresarial y la intención emprendedora en estudiantes de las Universidades Públicas de la Macro región Sur del Perú. Revista Innova Educación, 2(1), 177-192. https://doi.org/10.35622/j. $\underline{\text { rie.2020.01.011 }}$

Fernández, M y Quintero, N. (2017). Liderazgo transformacional y transaccional en emprendedores venezolanos. Revista Venezolana de Gerencia, 22(77), 56-74. http:// www.redalyc.org/articulo.oa? $\mathrm{id}=29051457005$

Frías-Navarro, D. (2021). Apuntes de consistencia interna de las puntuaciones de un instrumento de medida. Universidad de Valencia. España. Disponible en: https://www. uv.es/friasnav/AlfaCronbach.pdf

Gínez-Silva, M. J., Astorga, C. M., \& Urchaga-Litago, J. D. (2019). Resiliencia psicológica a través de la edad y el sexo. Revista INFAD de Psicología. International Journal of Developmental and Educational Psychology., 4(1), 85-94. https://doi.org/10.17060/ IJODAEP.2019.N1.V4.1513

González, A., Morales, A. (2012). El Lado B del emprendimiento: casos chilenos de emprendimientos fallidos y su aplicación en el aula". Repositorio académico de la Universidad de Chile. http://www.tesis.uchile.cl/tesis/uchile/2012/ecgonzalez_g/html/ index-frames.html189

Guajardo, E. S., Durán, C., Escalera, M., Mora, B., Pacheco, Á., \& de los Ángeles Pérez, M. (2018). Discapacidad motora y Resiliencia en adultos. En Estudios del de- 
sarrollo humano y socioambiental, 236-252. https://dialnet.unirioja.es/servlet/ articulo? codigo $=6676037$

Hernández-López, D. M., Moncada-Toro, J. F., \& Henao-Colorado, L. C. (2018). Intención emprendedora de los empleados del sector privado de la ciudad de Medellín y su Área Metropolitana. Revista CEA, 4(8), 13-33. https://doi.org/10.22430/24223182.1045

Hooper, D., Coughlan, J., and Mullen, M. R. (2008). Structural Equation Modelling: Guidelines for Determining Model Fit. Electronic Journal of Business Research Methods, 6(1), 53-60.

Hu, L.-t., \& Bentler, P. M. (1999). Cutoff criteria for fit indexes in covariance structure analysis: Conventional criteria versus new alternatives. Structural Equation Modeling, 6(1), 1-55. https://doi.org/10.1080/10705519909540118

Kharrazi, A. (2018). Resilience. Encyclopedia of Ecology, 15(2), 414-418. https://doi. org/10.1016/B978-0-12-409548-9.10751-1

Koch-Tovar, J. (2012). Manual del empresario exitoso. Recuperado de https://www.yumpu.com/es/document/read/40813646/manual-del-empresario-exitoso-biblioteca

Krueger, N. F., Reilly, M. D., \& Carsrud, A. L. (2000). Competing models of entrepreneurial intentions. Journal of Business Venturing, 15(5-6), 411-432. https://doi. org/10.1016/s0883-9026(98)00033-0

Lee, S. H., \& Wong, P. K. (2004). An exploratory study of technopreneurial intentions: a career anchor perspective. Journal of Business Venturing, 19(1), 7-28. https://doi. org/10.1016/s0883-9026(02)00112-X

Liñán, F., \& Chen, Y.-W. (2006). Testing the entrepreneurial intention model on a twocountry sample. Documents de Treball, 7(6). https://www.researchgate.net/publication/28117836 Testing the Entrepreneurial_Intention_Model_on a Two-Country Sample

Monjarás, A., Bazán, A., Pacheco, Z., Rivera, J., Zamarripa, J y Cuevas, C. (2019). Diseños de investigación. Educación y Salud: Boletín Científico Instituto de Ciencias de la Salud Universidad Autónoma del Estado de Hidalgo, 15, 119-122. https://doi. org/10.29057/icsa.v8i15.4908

Parra, M., Rubio, G., \& López, L. M. (2017). Emprendimiento y creatividad: Aspectos esenciales para crear empresa. Bogotá: Ecoe Ediciones.

Pérez, D. C., \& Ziglio, E. (2020). Fortaleciendo la resiliencia en tiempos de la COVID-19: una prioridad para la salud y para el progreso hacia los ODS. Revista Iberoamericana De Bioética, (14), 01-11. https://doi.org/10.14422/rib.i14.y2020.004

Romero, R. (2010). Emprendimiento y cultura para la perdurabilidad empresarial. Licenciatura. Universidad del Rosario. https://repository.urosario.edu.co/bitstream/handle /10336/2057/;jsessionid=CF988D728 0CCB42E4D3EDC01D96EACF0?sequence=1

Saavedra, E. (2011). La resiliencia desde una mirada post racionalista: Dos historias de vida. Saarbrücken: Académica Española 
Schneider, B. (2007). Resiliencia: Cómo construir empresas exitosas en contextos de inestabilidad. Bogotá, Colombia.

Shapero, A. and Sokol, L. (1982). The social dimensions of entrepreneurship. Encyclopaedia of entrepreneurship, 72-90.

Silva, G. S., Cordero, C. Z., \& Urdanivia, J. S. (2018). La resiliencia: un factor decisivo para el crecimiento y mejora de las organizaciones. Gestión en el tercer milenio, 20(39), 13-24.

Sivo, S. A., Fan, X., Witta, E. L., \& Willse, J. T. (2006). The Search for “Optimal” Cutoff Properties: Fit Index Criteria in Structural Equation Modeling. The Journal of Experimental Education, 74(3), 267-288. http://dx.doi.org/10.3200/JEXE.74.3.267-288

Sulivan. O, et al. (2020). Contributory factors to the evolution of the concept and practice of informed consent in clinical research: A narrative review. Contemporary Clinical Trials Communications. https://doi.org/10.1016/j.conctc.2020.100634

The jamovi project (2020). Jamovi. (Versión 1.2) [Computer Software].https://www.jamovi.org

Uriarte Arciniega, J. D. D. (2005). La resiliencia. Una nueva perspectiva en psicopatología del desarrollo Resilience. A new perspective into developmental psychopathology. Revista de Psicodidáctica, 10, 61-80.

Villamar, F. H. L., \& Harold, F. (2015). La resiliencia: su aplicación en el sector empresarial. Revista Contribuciones a la Economía.

Zúñiga, S. (2017). Liderazgo transformacional y resiliencia en el personal administrativo de una universidad privada de Chiclayo, 2017. Repositorio institucional de la Universidad César Vallejo. 